Case report

\title{
Clinical and pathologic features of Philadelphia chromosome-like acute lymphoblastic leukemia in a pediatric patient
}

\author{
Amrou Abdelkader, Horatiu Olteanu* \\ Department of Pathology, Medical College of Wisconsin, Milwaukee, USA
}

\begin{abstract}
Philadelphia chromosome-like acute lymphoblastic leukemia (ALL), also referred to as $B C R$ - $A B L 1$-like $A L L$, is a high-risk subset of $B$-lymphoblastic leukemia/lymphoma that shares a significant gene expression profile with the Philadelphia-positive ALL and generally has a less favorable outcome, compared to other ALL subtypes. Because of the clinical importance of these cases, Philadelphia-like ALL has been included as a provisional entity in the 2016 WHO classification of hematolymphoid neoplasms. Here we report the clinical and pathologic findings in a patient with Philadelphia-like ALL, with emphasis on the practical diagnostic and prognostic aspects of this pediatric malignancy.
\end{abstract}

Keywords: acute lymphoblastic leukemia; Philadelphia chromosome; BCR-ABL1; CRLF2

\section{Introduction}

Acute lymphoblastic leukemia (ALL) is the most common childhood malignancy, and remains a leading cause of death in pediatric patients. While current 5-year overall survival rates in children are $85 \%$ or higher, the survival following relapse is significantly decreased. From a biological standpoint, ALL is a heterogeneous entity comprised of multiple subsets with distinct genetic alterations, clinical features, and variable response to therapy [1].

$B C R-A B L$ 1-like

B-lymphoblastic leukemia/lymphoma (B-ALL), also known as Philadelphia $(\mathrm{Ph})$ chromosome-like ALL, is a neoplasm of lymphoblasts committed to the Blineage, that lack the $B C R-A B L 1$ translocation, but show a pattern of gene expression very

Received: March 2018; Accepted after review: May 2018; Published: June 2018.

${ }^{*}$ Corresponding author: Horatiu Olteanu, MD, PhD, Division of Hematopathology, Department of Pathology and Laboratory Medicine, Mayo Clinic, 200 First Street SW, Rochester, MN 55905.

Email : olteanu.horatiu@mavo.edu similar to that seen in ALL with $B C R-A B L 1$ [2]. Leukemias with these genetic profiles frequently demonstrate translocations of other tyrosine kinases, or alternatively have translocations involving the CRLF2 gene. Less often, they may show rearrangements leading to truncation and activation of EPOR. From a practical standpoint, it may be difficult to both identify such patients without a complex laboratory set-up, and it may be difficult to screen cases of newly diagnosed B-ALL to determine which patients would need such analysis. Because of its clinical importance, Ph-like ALL is recognized as a provisional entity in the 2016 World Health Organization classification of myeloid neoplasms and acute leukemias, and recent studies have helped define the genomic landscape of this entity. In addition, preclinical studies have demonstrated the efficacy of targeted inhibitors of relevant signaling pathways in specific Ph-like ALL subgroups, and precision medicine clinical trials have been initiated for this high-risk ALL subset [2].

In this case report, we describe a pediatric patient diagnosed with Ph-like B-ALL. We 
summarize the clinical, morphologic, immunophenotypic, cytogenetic and molecular findings in this patient, and discuss them in the context of our current understanding of this entity.

\section{Case report}

The patient is a 17 years-old, previously healthy female who presented to the emergency department with a 1-week history of headaches, diffuse myalgias, night sweats, nausea and emesis, nose bleeds and teacolored urine. Her past medical history was significant for polycystic ovary syndrome, and her family history included a great aunt with leukemia, and a grandmother with ovarian cancer. Physical examination revealed bulky bilateral cervical lymphadenopathy, and she was presumed to have infectious mononucleosis. Initial laboratory investigation performed in the emergency department revealed a mild elevation of liver function enzymes and a negative Monospot test. In addition, testing for viral hepatitis $A, B$ and $C$ was also negative. A complete blood count was performed and showed a WBC count of $8.2 \times 10^{9} / \mathrm{L}$, hemoglobin $11.8 \mathrm{~g} / \mathrm{dL}$ and platelet count of $43 \times 10^{9} / \mathrm{L}$. The differential count revealed $25 \%$ blasts, $12 \%$ segmented neutrophils, $61 \%$ lymphocytes, and $2 \%$ monocytes.

Because of the findings of anemia, thrombocytopenia, and circulating blasts, the patient was transferred to the Pediatric Hematology/Oncology unit for further workup, which included peripheral blood flow cytometry and bone marrow biopsy. The bone marrow aspirate smears showed numerous, hypercellular particles. Blasts comprised $80 \%$ of nucleated cells and consisted of mediumsized cells with high nuclear-to-cytoplasmic ratio, mild nuclear irregularities, coarsely dispersed chromatin, inconspicuous nucleoli, and small to moderate amount of deeply basophilic cytoplasm with occasional vacuoles. The core biopsy was $100 \%$ cellular and demonstrated sheets of blasts with similar morphology to that described in the aspirate smear (Figure 1). Peripheral blood flow cytometric immunophenotyping revealed a $40 \%$ population of aberrant B-lymphoblasts, that were CD45 (-), CD10 (+), CD22 (+), CD20 variably (+), CD19 (+), HLA-DR variably (+), CD34 partial (+), CD56 partial (+), Tdt (+), and negative for the remaining lymphoid and myeloid markers tested, including CD14, CD5, CD7, CD33, CD13, CD3, CD4, CD8, CD117, CD16, and MPO (Figure 2). Conventional cytogenetic analysis performed on bone marrow aspirate demonstrated a normal female karyotype $46, X X$, in 20 out of 20 cells examined. Fluorescence in situ hybridization (FISH) did not detect a $t(9 ; 22)$ translocation in 200 cells analyzed, but was positive for a deletion $12 p$ (ETV6) in $72 \%$ of nuclei. In addition, a CRLF2 rearrangement was observed in $51 \%$ of cells analyzed, and an extra $\operatorname{lgH}$ signal was present in $57 \%$ of cells, likely due to a translocation with CRLF2. Molecular analysis was negative for a JAK2 V617 mutation. Additional low-density microarray (LDA) testing performed at the University of New Mexico was positive for a Ph-like ALL signature, with high CRLF2 expression, and negative for the P2RY8CRLF2 translocation.

The patient underwent induction chemotherapy according to the CALGB 10403 protocol [3]. A bone marrow aspirate on day 15 of induction chemotherapy showed a hypocellular marrow with minimal residual BALL by flow cytometry. A repeat bone marrow evaluation at the time of peripheral blood count recovery (on day 28 after initiation of induction chemotherapy) demonstrated a hypercellular marrow with increased blasts, consistent with persistent disease. The patient subsequently failed re-induction chemotherapy and is currently being worked up for anti-CD19 chimeric antigen receptor T-cell (CAR-T) therapy. 

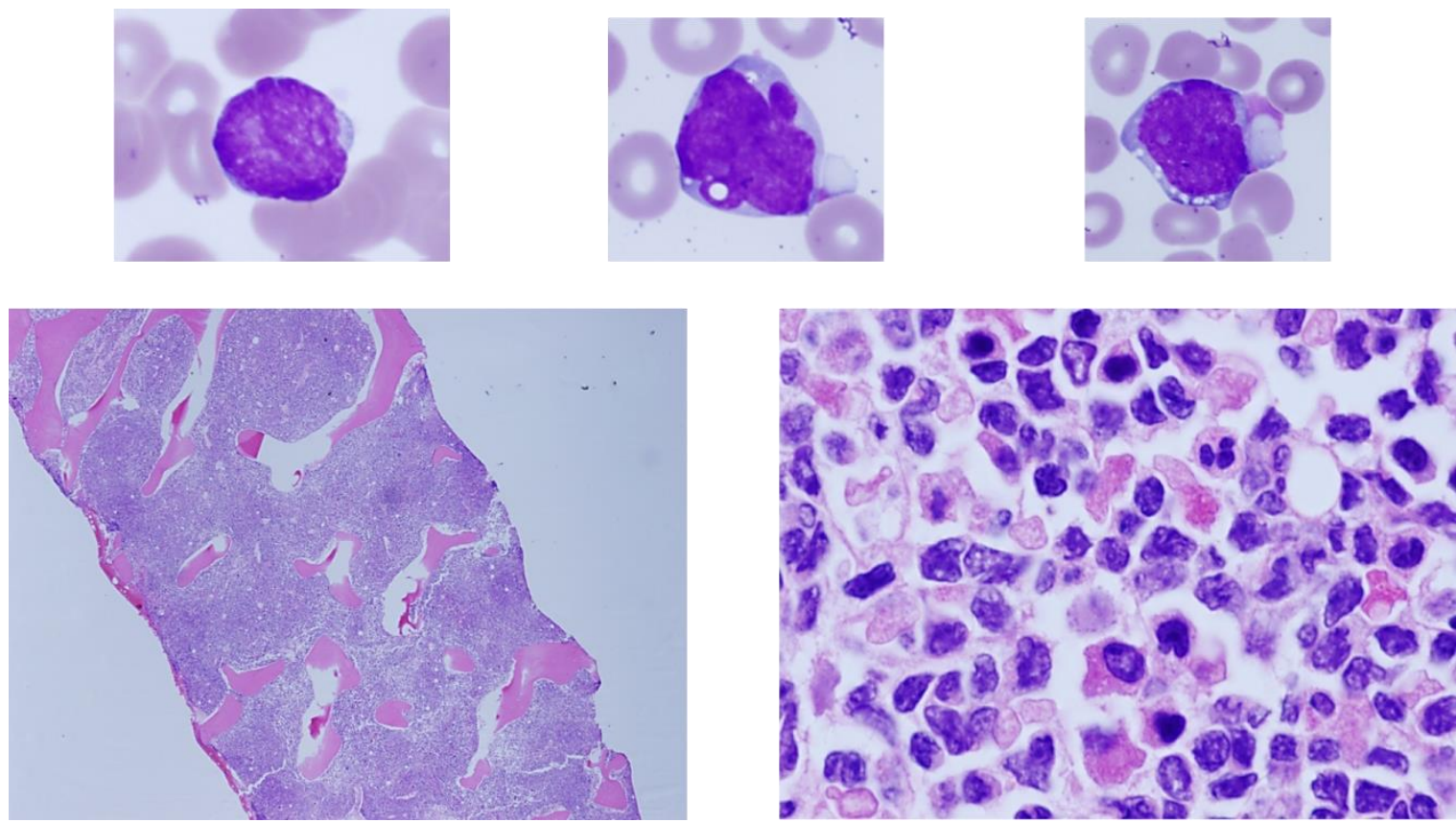

Fig. 1. Bone marrow morphologic findings at the time of B-lymphoblastic leukemia/lymphoma diagnosis : blasts comprised $80 \%$ of the bone marrow aspirate nucleated cells and consisted of medium-sized cells with high nuclear-to-cytoplasmic ratio, mild nuclear irregularities, coarsely dispersed chromatin, inconspicuous nucleoli, and small to moderate amount of deeply basophilic cytoplasm with occasional vacuoles. The core biopsy was $100 \%$ cellular and demonstrated sheets of blasts with similar morphology to that described in the aspirate smear.
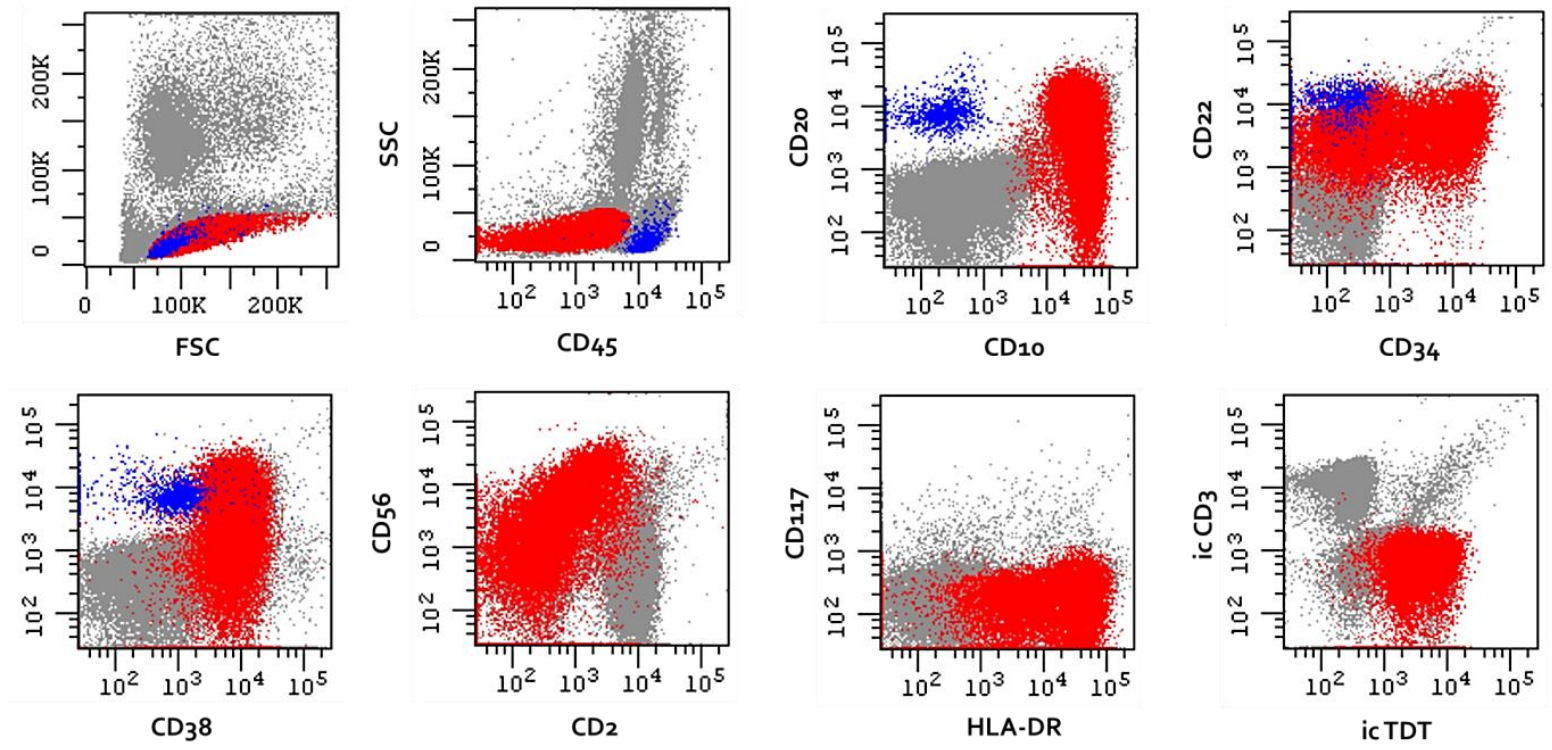

Fig. 2. Peripheral blood flow cytometric findings at the time of B-lymphoblastic leukemia/lymphoma diagnosis: immunophenotyping revealed a $40 \%$ population of aberrant B-lymphoblasts, that were CD45 (-), CD10 (+), CD22 (+), CD20 variably (+), CD19 (+), HLA-DR variably (+), CD34 partial (+), CD56 partial (+), Tdt (+), and negative for the remaining lymphoid and myeloid markers tested, including CD14, CD5, CD7, CD33, CD13, CD3, CD4, CD8, CD117, CD16, and MPO. 


\section{Discussion}

Herein we present a patient with $\mathrm{Ph}$-like B$A L L$, including the typical laboratory workup associated with this diagnosis and illustrating the generally poor prognosis associated with this entity. Ph-like B-ALL is relatively common, with a prevalence of $10-25 \%$ in patients with ALL. The highest frequency has been described in adults, adolescents and children with United States National Cancer Institute ( $\mathrm{NCl}$ ) high-risk ALL, while occurring less often in children with standard-risk ALL. The frequency of certain genomic alteration varies with certain genetic and ethnic backgrounds. For example, individuals with Native American genetic ancestry and Hispanics have a higher frequency of IGH/CRLF2 translocations, while CRLF2 translocations are very common in children with Down syndrome [2, 4].

The clinical and morphologic features in patients diagnosed with Ph-like B-ALL are in general similar to those seen in other types of ALL, although presentation with an elevated white blood cell count is one of the high-risk clinical features that tend to be more common in this subset. The blast immunophenotype is also typical to that of other types of B-ALL, including expression of CD19 and CD10. The evaluation of CRLF2 protein expression by flow cytometry has diagnostic utility, as a subset of cases with CRLF2 translocations demonstrate very high levels of CRLF2 protein expression on blasts, and thus can be used as a screening tool for this type of translocations. In contrast, there is no specific immunophenotypic signature associated with translocation involving the EPOR gene or other tyrosine kinases [2].

Ph-like/BCR-ABL1-like B-ALL was independently described by two groups as a subtype of ALL that had a gene expression profile very similar to that of Ph-like ALL, a high frequency of deletions of IKZF1 (which codes for the lymphoid transcription factor, IKAROS), and of other lymphoid transcription factor genes, such as $P A X 5$ and $E B F 1$, and an overall poor survival $[5,6]$. The analytic approaches and probes set used by Mullighan and colleagues from the Children's Oncology Group (COG)/St. Jude Children's Research
Hospital (SJCRH) were somewhat different than those employed by the Dutch group of Den Boer and colleagues, which led to significant overlap, but also to some discordant case assignment between Ph-like B-ALL cases (COG/SJCRH definition) and $B C R-A B L 1$-like B-ALL cases (as defined by the group from the Netherlands). Furthermore, of the 110 probe set included in the Dutch $B C R$ - $A B L$ 1-like B-ALL signature and the 225 probe set for the COG/SJCRH Ph-like ALL signature, only 9 of these (recognizing 7 genes) are shared in the two panels. Of note, none of these 9 probes are included in the 8gene low density array (LDA) Ph-like ALL signature developed at the University of New Mexico and used by the COG to identify patients with Ph-like ALL $[7,8]$. However, from a practical standpoint, it is important to keep in mind that specific underlying genomic lesions may potentially serve as clinically actionable therapeutic targets, rather than the Ph-like / $B C R-A B L 1$-like status itself.

As such, Ph-like ALL may show various types of chromosomal alterations, involving many different gene translocations and various partners. For example, about $50 \%$ of cases show CRLF2 gene rearrangements, usually associated with an interstitial deletion of the PAR1 gene family, which juxtaposes CRLF2 to the promoter of the P2RY8 gene. Another translocation partner is the immunoglobulin heavy chain gene, $I G H$, which can also be involved in translocations with EPOR. Another set of patients demonstrate translocation involving tyrosine kinases, such as $A B L 1$ (with genes other than $B C R$ ), $A B L 2, P D G F R B$, NTRK3, TYK2, CSF1R, and JAK2. CRLF2 rearrangements and kinase translocation appears to be mutually exclusive, as they rarely coexist in the same patient. While some of these translocations may be detected by standard cytogenetic analysis (karyotype), CRLF2 interstitial deletions are often cryptic and require other diagnostic methodologies. Of note, about half of the cases of Ph-like ALL with CRLF2 rearrangements have also mutations of JAK2 or JAK1 [2, 4].

Because of this fairly complex genomic landscape of Ph-like ALL, there is no single method or universally accepted algorithm for effectively screening and identifying potential 
patients with this leukemia. Potential methods include: (i) low density array (LDA)analysis, which is currently used in the research/clinical trial setting by the COG to initially screen for $\mathrm{Ph}$-like signature; (ii) FISH analysis with break apart probes for known kinase genes; while this is a potentially simple and cost effective test, it is limited in scope; (iii) RT-PCR may screen for or confirm known gene fusions; (iv) capture-based RNA sequencing (RNAseq); and (v) next-generation sequencing (NGS); the latter two methods have the advantage of flexibility and potential for covering an increased number of potential target genes, but also with a significantly higher price tag.

For example, the current Ph-like ALL genetic testing algorithm used by the COG employs the LDA 8-gene panel to screen, followed by a series of multiplex RT-PCR assays, $\mathrm{FISH}$, and DNA sequencing to identify the underlying genomic alterations. Based on those results, patients with Ph-like ALL and $A B L$-class fusions have the tyrosine kinase inhibitor, dasatinib added to chemotherapy starting with the second month of treatment. In contrast, high risk Ph-like B-ALL patients with CRLF2/JAK pathway lesions are eligible for the AALL1521 trial which combines postinduction therapy with ruxolitinib [8]. Another approach, also for clinical trial purposes, taken by the SJCRH group involves performing RNAseq in all patients enrolled in their Total XVII study (ClinicalTrials.gov Identifier: NCT03117751).

The testing algorithm developed by the MD Anderson trial for Ph-like B-ALL involves screening all newly diagnosed or relapsed BALL patients for CRLF2 expression by flow cytometry; BCR-ABL1 translocation by FISH/PCR; and for gene copy number analysis by MLPA/SNP microarray. Based on those initial screening results, patients with high level of CRLF2 by flow cytometry undergo confirmatory testing by CRLF2 metaphase FISH and for targeted JAK2 mutations, while those with negative CRLF2 flow cytometry results are further tested by FISH for $A B L 1$, $A B L 2, P D G F R B, C S F 1 R$, JAK2, and EPOR rearrangements. If initial screening is negative, additional testing for a PCR-based fusion gene panel is available [8].
Finally, a Ph-like ALL 6-gene panel (including CRLF2, $A B L 1, A B L 2, \quad P 2 R Y 8$, $P D G F R B$, and JAK2) is commercially available from the Mayo Medical Laboratories by FISH (https://www.mayomedicallaboratories.com/tes t-catalog/Overview/35256).

\section{Conclusion}

In summary, Ph-like B-ALL comprises a significant subset of patients with ALL, and has been associated with inferior outcomes in most studies. Overall, these patients do not only have a poor prognosis, but also have a higher risk for being positive for minimal residual disease (MRD), and it is unclear to what extent the outcome is being independently impacted by the MRD status. $\mathrm{Ph}$-like B-ALL is characterized by kinase rearrangements, of which CRLF2 alterations are the most prevalent, and often associate with JAK1 or JAK2 mutations. In patients with this type of B-ALL, screening for targetable kinase alterations is the most critical aspect of the diagnostic algorithm, as demonstrated in the subset of children with PDGFRB translocations and primary resistance to induction chemotherapy [9]. These patients have shown significant responses to treatment with tyrosine kinase inhibitors such as imatinib and dasatinib, and this hypothesis is further tested in clinical trials targeting patients with ABL-class fusions, with the goal of improving the poor outcome. From a practical standpoint, there are multiple testing modalities available, although an algorithm for the optimal detection of patients with Ph-like B-ALL is likely dependent on individual patient population characteristics and available resources.

\section{Consent}

Written informed consent was obtained from the patient's parents for publication of this case report.

\section{Competing interests}

The authors declare that they have no competing interests. 


\section{References}

1. Hunger SP, Mullighan CG. Redefining ALL classification: toward detecting high-risk ALL and implementing precision medicine. Blood 2015; 125(26):3977-3987.

2. Borowitz MJ, Chan JKC, Downing JR, LeBeau MM, Arber DA. B-lymphoblastic leukemia/lymphoma, $B C R-A B L$ 1-like. In: Swerdlow SH, Campo E, Harris NL, Jaffe ES, Pileri SA, Stein $\mathrm{H}$, et al., editors. WHO Classification of Tumors of Hematopoietic and Lymphoid Tissues (revised $4^{\text {th }}$ edition). Lyon: IARC; 2017. p. 308.

3. Ribera JM. Advances in acute lymphoblastic leukemia in adults. Curr Opin Oncol 2011; 23(6):692-699.

4. Tasian SK, Loh ML, Hunger SP. Philadelphia chromosome-like acute lymphoblastic leukemia. Blood 2017; 130(19):2064-2072.

5. Mullighan CG, Su X, Zhang J, Radtke I, Phillips LA, Miller CB, et al. Deletion of IKZF1 and prognosis in acute lymphoblastic leukemia. $\mathrm{N}$ Engl J Med 2009; 360(5):470-480.

6. Den Boer $M L$, van Slegtenhorst $M$, De Menezes RX, Cheok MH, Buijs-Gladdines JG,
Peters ST, et al. A subtype of childhood acute lymphoblastic leukaemia with poor treatment outcome: a genome-wide classification study. Lancet Oncol 2009; 10(2):125-134.

7. Boer JM, Marchante JR, Evans WE, Horstmann MA, Escherich G, Pieters R, et al. BCR-ABL1-like cases in pediatric acute lymphoblastic leukemia: a comparison between DCOG/Erasmus $\mathrm{MC}$ and COG/St. Jude signatures. Haematologica 2015; 100(9):e354e357.

8. Reshmi SC, Harvey RC, Roberts KG, Stonerock $E$, Smith $A$, Jenkins $H$, et al. Targetable kinase gene fusions in high-risk BALL: a study from the Children's Oncology Group. Blood 2017; 129(25):3352-3361.

9. Weston BW, Hayden MA, Roberts KG, Bowyer S, Hsu J, Fedoriw G, et al. Tyrosine kinase inhibitor therapy induces remission in a patient with refractory EBF1-PDGFRB-positive acute lymphoblastic leukemia. J Clin Oncol 2013; 31(25):e413-e416. 